\title{
A Nonaxisymmetric Solution of Einstein's Equations Featuring Pure Radiation from a Rotating Source
}

\author{
William Davidson ${ }^{1,2}$ \\ ${ }^{1}$ Mathematics and Statistics Department, University of Otago, Dunedin, New Zealand \\ ${ }^{2} 21$ Rowbank Way, Loughborough, Leicestershire LE11 4AJ, UK
}

Correspondence should be addressed to William Davidson, wdav295@btinternet.com

Received 7 January 2012; Accepted 8 February 2012

Academic Editors: H. Dehnen and N. Fornengo

Copyright ( 2012 William Davidson. This is an open access article distributed under the Creative Commons Attribution License, which permits unrestricted use, distribution, and reproduction in any medium, provided the original work is properly cited.

\begin{abstract}
A special nonaxisymmetric solution of Einstein's equations is derived, representing pure radiation from a rotating isolated source. The spacetime is assumed to be algebraically special having a multiple null eigenvector of the Weyl tensor forming a geodesic, shear-free, diverging, and twisting congruence $\mathbf{k}$. Employing a complex null tetrad involving the vector $\mathbf{k}$, the Ricci tensor, density of the radiation, divergence, and twist are calculated for the derived metric. A particular (nonaxisymmetric) subcase is shown to be flat at infinity and to contain the axisymmetric radiating Kerr metric, derived by Kramer and separately by Vaidya and Patel, as a special case. The spacetime is of Petrov type II and without Killing vectors.
\end{abstract}

\section{Introduction}

In this paper, we present a special solution to Einstein's equations involving pure radiation. Pure radiation is characterised by an energy-momentum tensor of the form

$$
T_{a b}=\phi^{2} k_{a} k_{b},
$$

where $\mathbf{k}$ is a null vector. The incoherent radiation is propagated along the $\mathbf{k}$ lines and $\phi^{2}$ is its energy density, which must, therefore, be positive. For our purpose, it will be assumed that the $\mathbf{k}$ vector field forms a geodesic, shearfree congruence, that $\mathbf{k}$ is a multiple eigenvector of the Weyl tensor, and that the metric is algebraically special. In addition, the congruence will be taken to be diverging and twisting. The consequences of these assumptions will be detailed in Section 2. In particular, they lead to a form of the metric originally derived by I. Robinson and J. R. Robinson [1] and Robinson et al. [2].

To obtain solutions of Einstein's equations representing pure radiation, authors have in the main assumed some form of symmetry. An early one of spherical symmetry by Vaidya [3] featured pure radiation from a central Schwarzschild body, with the mass being consequently a function $m(u)$ of the time coordinate $u$. This was followed by the axisymmetric radiating Kerr metric by Kramer [4], and also a different version of this by Vaidya and Patel [5], where in the latter setting a certain parameter to zero regained the Kerr metric in its original form [6]. These two versions represent pure radiation from a rotating central source. Herlt [7] found the complete solution for pure radiation in axisymmetric KerrSchild metrics (cf. Debney et al. [8]).

More recently, pure radiation solutions have been derived by exploiting possible symmetry groups in the hyperspace $r=$ const (see (8)). These authors [9-12] employed the theory of Cauchy-Riemann structures (see, e.g., Robinson and Trautman [13]). An axisymmetric solution of some generality, involving pure radiation from a rotating source, has been derived up to a linear second-order ordinary differential equation by Kramer and Hähner [14].

A valuable method of generating algebraically special pure radiation fields from vacuum cases was devised by Stephani, thereby obtaining some explicit axisymmetric solutions [15]. In this paper, we shall employ Stephani's device but will generate a nonaxisymmetric solution, which has the radiating Kerr metric as a special case. If a star can catastrophically collapse through its event horizon and if its ultimate state is a rotating axisymmetric Kerr black hole, then the time-dependent solution given here may be interpreted as a theoretical example of a nonaxisymmetric stage in that process. 


\section{The Basic Framework}

Because of our assumptions in Section 1, we can write the following as properties of the $\mathbf{k}$ congruence:

(i) since $\mathbf{k}$ is geodesic, $\kappa=0 \Rightarrow k_{[a} k_{b]_{;}} k^{c}=0$, or via an affine parameter $k_{a ; b} k^{b}=0$;

(ii) $\mathbf{k}$ is shear-free:

$$
\sigma=0 \Longrightarrow k_{(a ; b)} k^{a ; b}-\frac{1}{2}\left(k_{; a}^{a}\right)^{2}=0
$$

(iii) $\mathbf{k}$ is diverging and twisting: $\Theta=(1 / 2) k_{; a}^{a}>0, \omega^{2}=$ $(1 / 2) k_{[a ; b]} k^{a ; b}>0$

(iv) $\mathbf{k}$ is a multiple Weyl eigenvector: $\psi_{0}=\psi_{1}=0$.

We shall refer the spacetime to a complex null tetrad $\mathbf{m}$, mbar, $\mathbf{n}$, and $\mathbf{k}$ with labels 1, 2, 3, and 4, respectively, with the fourth member being the $\mathbf{k}$ of the above context. In relation to this tetrad, in order to display the form of the metric we adopt coordinates $x^{i}=x, y, r$, and $u$ for $i=1,2,3$, and 4 , respectively. The spacelike coordinate $x$ is complex, $y$ its conjugate, $r$ an affine parameter along the $\mathbf{k}$ lines, and $u$ a retarded time. The Newman-Penrose spin coefficients $\kappa, \sigma$ and $\rho=-(\Theta+i \omega)$ are expressible in terms of the tetrad by the relations:

$$
\begin{gathered}
\kappa=-k_{a ; b} m^{a} k^{b}=0, \quad \sigma=-k_{a ; b} m^{a} m^{b}=0, \\
\rho=-(\Theta+i \omega)=-k_{a ; b} m^{a} \bar{m}^{b} \neq 0 .
\end{gathered}
$$

It now becomes possible to write the metric in the following form $[1,2]$ (we use mainly the notation of [16]):

$$
\mathrm{d} s^{2}=2 \omega^{1} \omega^{2}-2 \omega^{3} \omega^{4}
$$

where

$$
\begin{aligned}
& \omega^{1}=-\frac{\mathrm{d} x}{P \bar{\rho}}, \\
& \omega^{2}=-\frac{\mathrm{d} y}{P \rho}, \\
& \omega^{3}=\mathrm{d} u+L \mathrm{~d} x+\bar{L} \mathrm{~d} y, \\
& \omega^{4}=\mathrm{d} r+W \mathrm{~d} x+\bar{W} \mathrm{~d} y+H \omega^{3} .
\end{aligned}
$$

The dual frame is then, relative to the $x^{i}$ system,

$$
\begin{gathered}
m^{i}=(-P \bar{\rho}, 0, P W \bar{\rho}, P L \bar{\rho}), \\
\bar{m}^{i}=(0,-P \rho, P \bar{W} \rho, P \bar{L} \rho), \\
n^{i}=(0,0,-H, 1), \\
k^{i}=(0,0,1,0) .
\end{gathered}
$$

Here

$$
\begin{gathered}
\rho=-\frac{1}{(r+i \Sigma)}, \\
\Sigma=-\frac{i P^{2}(\bar{\partial} L-\partial \bar{L})}{2}, \\
W=\frac{L_{, u}}{\rho}+i \partial \Sigma, \\
\partial \equiv \partial_{x}-L \partial_{u},
\end{gathered}
$$

and the metric will take the following form:

$$
\begin{aligned}
\mathrm{d} s^{2}=-2\{ & H \mathrm{~d} u^{2}+(W+2 H L) \mathrm{d} u \mathrm{~d} x+(\bar{W}+2 H \bar{L}) \mathrm{d} u \mathrm{~d} y \\
& +\mathrm{d} u \mathrm{~d} r+L \mathrm{~d} x \mathrm{~d} r+\bar{L} \mathrm{~d} y \mathrm{~d} r \\
& +\left(L W+H L^{2}\right) \mathrm{d} x^{2}+\left(\overline{L W}+H \bar{L}^{2}\right) \mathrm{d} y^{2} \\
& \left.-\left(\frac{1}{\left(P^{2} \rho \bar{\rho}\right)}-L \bar{W}-\bar{L} W-2 H L \bar{L}\right) \mathrm{d} x \mathrm{~d} y\right\}
\end{aligned}
$$

In particular, the coefficient of $\mathrm{d} u^{2}$ is $-2 H$ where

$$
\begin{gathered}
H=\frac{K}{2}-r P^{-1} P_{, u}-\frac{(m r+M \Sigma)}{\left(r^{2}+\Sigma^{2}\right)}, \\
K=2 P^{2} \operatorname{Re}\left(\partial\left(P^{-1} \bar{\partial} P-\bar{L}_{, u}\right)\right),
\end{gathered}
$$

and $M$ will be defined by (11).

In these relations $P, K, m$, and $M$ are real functions, and $L$ is a complex function, of $x, y$, and $u$. $H$ is a real function and $W$ a complex function of $x, y, r$, and $u$. A subscript indicates partial differentiation.

For pure radiation, the two equations to be satisfied are (cf. [16])

$$
\begin{gathered}
\left(3 L_{, u}-\partial\right)(m+i M)=0 \\
M=\Sigma K+P^{2} \operatorname{Re}\left(\partial \bar{\partial} \Sigma-2 \bar{L}_{, u} \partial \Sigma-\Sigma \partial_{u} \partial \bar{L}\right) .
\end{gathered}
$$

Then $\phi^{2}$ is given by the equation

$$
P^{4}\left(\partial-2 L_{, u}+2 P^{-1} \partial P\right) \partial C-P^{3}\left(P^{-3}(m+i M)\right)_{, u}=\frac{\phi^{2}}{(2 \rho \bar{\rho})},
$$

where

$$
C=\bar{\partial}\left(P^{-1} \bar{\partial} P-\bar{L}_{, u}\right)+\left(P^{-1} \bar{\partial} P-\bar{L}_{, u}\right)^{2}
$$

\section{A Nonaxisymmetric Pure Radiation Rotation Field}

Referring to (1), we have from the energy equation $T^{a b}{ }_{; b}=$ 0 , using the properties listed in (2), namely, $k_{a ; b} k^{b}=0$, 
$\Theta=(1 / 2) k_{; a}^{a}=-(1 / 2)(\rho+\bar{\rho})$, and noting from (6) that $\mathbf{k}=\partial_{r}$, that $\phi^{2}$ has the form

$$
\phi^{2}=\psi(x, y, u) \rho \bar{\rho} .
$$

For the functions $L$ and $P$, we initially choose

$$
\begin{gathered}
P=P_{0}=a x y+b(x+y)+c, \\
L=L_{0}=-\frac{i\left(n_{0}+n_{1} y+n_{2} y^{2}\right)}{(a x y+b(x+y)+c)^{2}}, \\
m=m_{0},
\end{gathered}
$$

where $a, b, c, n_{0}, n_{1}, n_{2}$, and $m_{0}(>0)$ are real constants.

We now derive from the definitions in Section 2 that

$$
M=M_{0}=0 .
$$

Henceforth, we shall calculate tensor components relative to the complex null tetrad. In that frame, the metric coefficients are

$$
g_{a b}=\left(\begin{array}{cccc}
0 & 1 & 0 & 0 \\
1 & 0 & 0 & 0 \\
0 & 0 & 0 & -1 \\
0 & 0 & -1 & 0
\end{array}\right) .
$$

In particular, we find for the Ricci tensor

$$
R_{a b}=0 .
$$

We, therefore, have a vacuum spacetime. Accordingly, we shall now adopt the method of Stephani [15] to generate a pure radiation field.

Stephani's result can be stated: given $m=m_{0}(=$ const), $M_{0}(=0), L_{0}, P_{0}$ specifying an algebraically special vacuum solution to Einstein's equations, then if we change (only) $P$ from $P_{0}$ to $P_{0} A(x, y, u)$ and if (10) and (11) remain valid (with $M=M_{0}=0$ ), then the resultant spacetime represents a field of pure radiation.

For our case, starting with (15) except for the modification of $P$, we find that Stephani's conditions are satisfied if we adopt for $A(x, y, u)$ the value

$$
A(x, y, u)=u
$$

provided we set

$$
n_{2}=\frac{\left(b n_{1}-a n_{0}\right)}{c} .
$$

$\Sigma$ and $K$ will be altered accordingly in (7) and (9), with $L=L_{0}$ remaining unchanged.

We may verify that with the revised value of $P$ that $M=$ $M_{0}=0$, and that the equations for pure radiation, (10) and (11), are then satisfied.

Calculation of the Ricci tensor in the null tetrad yields the result:

$$
R_{a b}=\frac{6 m_{0}}{u} \rho \bar{\rho} \delta_{a}^{3} \delta_{b}^{3}
$$

It, therefore, follows from (1) and (21), or from (12), that $\phi^{2}=\left(6 m_{0} / u\right) \rho \bar{\rho}$. From the definition of $\rho$ in $(7)$, and also as $-(\Theta+i \omega)$, we obtain for the expansion and twist of the $\mathbf{k}$ congruence:

$$
\begin{aligned}
\Theta= & \frac{c^{2} r[a x y+b(x+y)+c]^{2}}{D}, \\
\begin{array}{c}
\omega= \\
-
\end{array} & u^{2} c[a x y+b(x+y)+c] \\
& \times\left\{2 n_{0}[a b x y+a c(x+y)+b c]\right. \\
& \left.\quad-n_{1}\left[\left(2 b^{2}-a c\right) x y+b c(x+y)+c^{2}\right]\right\} / D, \\
D= & c^{2} r^{2}[a x y+b(x+y)+c]^{2} \\
& +u^{4}\left\{2 n_{0}[a b x y+a c(x+y)+b c]\right. \\
& \left.\quad-n_{1}\left[\left(2 b^{2}-a c\right) x y+b c(x+y)+c^{2}\right]\right\}^{2} .
\end{aligned}
$$

For the energy density of the pure radiation, we can now write

$$
\phi^{2}=\frac{6 m_{0} c^{2}[a x y+b(x+y)+c]^{2}}{u D},
$$

which is $>0$, as required, and $\rightarrow 0$ as either $r$ or $u \rightarrow \infty$. Because of the radiation emission, the central isolated mass will steadily decrease.

The Ricci tensor being given by (21), we have the invariants:

$$
R=0, \quad R_{a b} R^{a b}=0 .
$$

\section{Behaviour at Infinity}

Our spacetime becomes axisymmetric if we set $b=0$. But if we go further and adopt a subset of our previous assignments:

$$
\begin{gathered}
b=0, \quad a=\frac{1}{2}, \quad c=1, \\
P(x, y, u)=\left(1+\frac{x y}{2}\right), \quad L(x, y)=-\frac{\text { in } y}{(1+x y / 2)^{2}},
\end{gathered}
$$

and then make the transformation

$$
\begin{aligned}
x=\sqrt{2} \tan \left(\frac{\theta}{2}\right) e^{i \xi}, & y=\sqrt{2} \tan \left(\frac{\theta}{2}\right) e^{-i \xi}, \\
r & =r, \quad u=t
\end{aligned}
$$


we obtain a form of the Kerr metric:

$$
\begin{aligned}
\mathrm{d} s^{2}= & \left(r^{2}+n^{2} \cos (\theta)^{2}\right) \mathrm{d} \theta^{2} \\
& +\sin (\theta)^{2}\left(r^{2}+n^{2}+\frac{2 n^{2} m_{0} r \sin (\theta)^{2}}{r^{2}+n^{2} \cos (\theta)^{2}}\right) \mathrm{d} \xi^{2} \\
& -2 n \sin (\theta)^{2} \mathrm{~d} \xi \mathrm{d} r-2 \mathrm{~d} r \mathrm{~d} t+\frac{4 n m_{0} r \sin (\theta)^{2}}{r^{2}+n^{2} \cos (\theta)^{2}} \mathrm{~d} \xi \mathrm{d} t \\
& -\left(1-\frac{2 m_{0} r}{r^{2}+n^{2} \cos (\theta)^{2}}\right) \mathrm{d} t^{2} .
\end{aligned}
$$

As is well known, the Kerr metric is flat at infinity. For any stationary metric associated with an isolated mass and which is flat at infinity, expansion in powers of $1 / r$ has the following form [17, page 452]:

$$
\begin{aligned}
\mathrm{d} s^{2}= & -\left(1-\frac{2 \bar{M}}{r}+O\left(\frac{1}{r^{2}}\right)\right) \mathrm{d} t^{2} \\
& -\left(4 \varepsilon_{j k m} S^{k} \frac{X^{m}}{r^{3}}+O\left(\frac{1}{r^{3}}\right)\right) \mathrm{d} X^{j} \mathrm{~d} t \\
+ & \left\{\left(1+\frac{2 \bar{M}}{r}+O\left(\frac{1}{r^{2}}\right)\right) \delta_{j k}\right. \\
& \left.+(\text { vanishing terms }) \delta_{j k}\right\} \mathrm{d} X^{j} \mathrm{~d} X^{k} .
\end{aligned}
$$

In (28), the $X^{j}$ are the coordinates of the ultimately flat spacetime,

$$
X=r \sin \theta \cos \xi, \quad Y=r \sin \theta \sin \xi, \quad Z=r \cos \theta
$$

and $S^{k}$ is the angular momentum vector of the source.

Comparing (28) with (27) (expanded in powers of $1 / r)$, we can confirm that the Kerr central mass is $\bar{M}=$ $m_{0}$. Moreover, as was proved by Cohen [18], the angular momentum of the Kerr mass is $n m_{0}$, so that the parameter $n$ in (25) is the angular momentum per unit mass.

If we now retain (25) except that we change $P$ to

$$
P(x, y, u)=\left(1+\frac{x y}{2}\right) u
$$

and follow through (26), then we have a form of the radiating Kerr metric (cf. $[4,5])$, namely,

$$
\begin{aligned}
\mathrm{d} s^{2}= & \frac{r^{2}+n^{2} t^{4} \cos (\theta)^{2}}{t^{2}} \mathrm{~d} \theta^{2}-4 n^{3} t \sin (\theta)^{3} \cos (\theta) \mathrm{d} \theta \mathrm{d} \xi \\
& +\sin (\theta)^{2}\left[n^{2} t^{2}+n^{4} \sin (\theta)^{4}+\frac{r^{2}}{t^{2}}\right. \\
& \left.+2 \frac{n^{2} r \sin (\theta)^{2}}{t}+2 \frac{n^{2} m_{0} r \sin (\theta)^{2}}{r^{2}+n^{2} t^{4} \cos (\theta)^{2}}\right] \mathrm{d} \xi^{2} \\
& -2 n \sin (\theta)^{2} \mathrm{~d} \xi \mathrm{d} r-4 n^{2} t \sin (\theta) \cos (\theta) \mathrm{d} \theta \mathrm{d} t+2 \sin (\theta)^{2} \\
& \times\left[n^{3} \sin (\theta)^{2}+2 \frac{n r}{t}\right. \\
& \left.+2 \frac{n m_{0} r}{r^{2}+n^{2} t^{4} \cos (\theta)^{2}}\right] \mathrm{d} \xi \mathrm{d} t-2 \mathrm{~d} r \mathrm{~d} t \\
& -\left[t^{2}-n^{2} \sin (\theta)^{2}-2 \frac{r}{t}-2 \frac{m_{0} r}{r^{2}+n^{2} t^{4} \cos (\theta)^{2}}\right] \mathrm{d} t^{2} .
\end{aligned}
$$

It may be shown for this metric that the central mass (of parameter $m_{0}$ ) has at epoch $u$ the value $m_{0} / u^{3}$ (see [14]), confirming its steady loss to radiation.

In the light of the foregoing, we see that our present solution is a nonaxisymmetric spacetime with a radiating rotating source. It has of course extra complication mathematically. But if we retain the expressions (26) for $x$ and $y$, then we find that the components of the Riemann tensor for our nonaxisymmetric case $\rightarrow 0$ as either $r$ or $u \rightarrow \infty$. In particular, if we adopt the following values:

$$
a=\frac{1}{2}, \quad c=1, \quad n_{1}=n, \quad n_{0}=2 b n \quad\left(n_{2}=0\right),
$$

and the assignment (26), then the components of the Riemann tensor $\rightarrow 0$ as $r \rightarrow \infty$ at rates varying from $r^{-2}$ to $r^{-4}$ and as $t \rightarrow \infty$ at rates between $t^{-4}$ and $t^{-8}$. Hence the associated metric is flat at infinity. We give its metric coefficients in the Appendix. There we see that if we set $b=0$ in this nonaxisymmetric pure radiation solution, then we obtain the axisymmetric radiating Kerr metric given at (31).

Our general metric, specified by (19) and (20), is of Petrov Type II and without Killing vectors.

\section{Conclusion}

A solution of Einstein's equations has been obtained, which features pure radiation from an isolated rotating source in a nonaxisymmetric environment. It contains the axisymmetric radiating Kerr metric as a special case.

An interesting aspect of the solution, therefore, is that its lack of symmetry suggests a possible theoretical model for the time-dependent configuration of the catastrophic collapse of a rotating star, during the approach to its final state of axial symmetry. 


\section{Appendix}

Here we give the metric coefficients for the (nonaxisymmetric) subcase:

$$
a=\frac{1}{2}, \quad c=1, \quad n_{1}=n, \quad n_{0}=2 b n \quad\left(n_{2}=0\right),
$$

having made the transformation

$$
\begin{aligned}
& x=\sqrt{2} \tan \left(\frac{\theta}{2}\right) e^{i \xi}, \quad y=\sqrt{2} \tan \left(\frac{\theta}{2}\right) e^{-i \xi}, \\
& r=r, \quad u=t, \\
& g_{\theta \theta}=\frac{512 b^{2} n^{2} \sin ^{2} \xi}{A^{4}(1+\cos \theta)^{2}}\left[2\left(1-2 b^{2}\right) t^{2}-\left(K-\frac{2 r}{t}-\frac{2 m_{0} r}{r^{2}+\Sigma^{2}}\right)\right] \\
& +\frac{1024 \sqrt{2} b n^{3} B t \sin \xi}{A^{5}(1+\cos \theta)^{2}}\left[\tan \left(\frac{\theta}{2}\right)+\sqrt{2} b \cos \xi\right] \\
& +\frac{16\left(r^{2} A^{2}+n^{2} B^{2} t^{4}\right)}{A^{4} t^{2}(1+\cos \theta)^{2}}, \\
& g_{\theta \xi}=-\frac{128 \sqrt{2} b n^{2} \tan (\theta / 2) \sin \xi}{A^{4}(1+\cos \theta)}\left[\tan \left(\frac{\theta}{2}\right)+\sqrt{2} b \cos \xi\right] \\
& \times\left[K-\frac{2 r}{t}-\frac{2 m_{0} r}{r^{2}+\Sigma^{2}}\right] \\
& +\frac{512 \sqrt{2} b\left(1-2 b^{2}\right) n^{2} t^{2} \tan (\theta / 2) \sin \xi}{A^{4}(1+\cos \theta)} \\
& \times\left[\tan \left(\frac{\theta}{2}\right)+\sqrt{2} b \cos \xi\right] \\
& +\frac{512 n^{3} B t \tan (\theta / 2)}{A^{5}(1+\cos \theta)} \\
& \times\left[2 b^{2}\left(2 \cos ^{2} \xi-1\right)+2 \sqrt{2} b \tan \left(\frac{\theta}{2}\right) \cos \xi+\tan ^{2}\left(\frac{\theta}{2}\right)\right], \\
& g_{\xi \xi}=-\frac{256 n^{2} \tan ^{2}(\theta / 2)}{A^{4}}\left[\tan \left(\frac{\theta}{2}\right)+\sqrt{2} b \cos \xi\right]^{2} \\
& \times\left[K-\frac{2 r}{t}-\frac{2 m_{0} r}{r^{2}+\Sigma^{2}}\right] \\
& +\frac{512\left(1-2 b^{2}\right) n^{2} t^{2} \tan ^{2}(\theta / 2)}{A^{4}}\left[\tan \left(\frac{\theta}{2}\right)+\sqrt{2} b \cos \xi\right]^{2} \\
& -\frac{1024 \sqrt{2} b n^{3} B t \tan ^{2}(\theta / 2) \sin \xi}{A^{5}}\left[\tan \left(\frac{\theta}{2}\right)+\sqrt{2} b \cos \xi\right] \\
& +\frac{16 \tan ^{2}(\theta / 2)}{A^{4} t^{2}}\left[r^{2} A^{2}+n^{2} t^{4} B^{2}\right] \\
& g_{\theta t}=\frac{16 \sqrt{2} b n \sin \xi}{A^{2}(1+\cos \theta)}\left[\left(1-2 b^{2}\right) t^{2}-\left(K-\frac{2 r}{t}-\frac{2 m_{0} r}{r^{2}+\Sigma^{2}}\right)\right] \\
& +\frac{32 n^{2} B t}{A^{3}(1+\cos \theta)}\left[\tan \left(\frac{\theta}{2}\right)+\sqrt{2} b \cos \xi\right]
\end{aligned}
$$

$$
\begin{aligned}
& g_{\xi t}=\frac{16 n \tan (\theta / 2)(\tan (\theta / 2)+\sqrt{2} b \cos \xi)}{A^{2}} \\
& \times\left[\left(1-2 b^{2}\right) t^{2}-\left(K-\frac{2 r}{t}-\frac{2 m_{0} r}{r^{2}+\Sigma^{2}}\right)\right] \\
& -\frac{32 \sqrt{2} b n^{2} B t}{A^{3}} \tan \left(\frac{\theta}{2}\right) \sin \xi, \\
& g_{\theta r}=-\frac{16 \sqrt{2} b n \sin \xi}{A^{2}(1+\cos \theta)}, \\
& g_{\xi r}=-\frac{16 n \tan (\theta / 2)}{A^{2}}\left(\tan \left(\frac{\theta}{2}\right)+\sqrt{2} b \cos \xi\right), \\
& g_{t r}=-1 \text {, } \\
& g_{t t}=-\left(K-\frac{2 r}{t}-\frac{2 m_{0} r}{r^{2}+\Sigma^{2}}\right), \\
& K=n^{2} \frac{(1+\cos \theta)\left[\left(1-2 b^{2}\right) \cos \theta-2 \sqrt{2} b \sin \theta \cos \xi-2 b^{2}-1\right]}{1+2 \sqrt{2} b \sin \theta \cos \xi+2 b^{2} \sin ^{2} \theta \cos ^{2} \xi} \\
& +\left(1-2 b^{2}\right) t^{2} \\
& A=\frac{4}{1+\cos \theta}[1+\sqrt{2} b \sin \theta \cos \xi] \text {, } \\
& B=\frac{4}{1+\cos \theta}\left[2 b^{2}(1+\cos \theta)+\sqrt{2} b \sin \theta \cos \xi-\cos \theta\right] \text {, } \\
& \Sigma=\frac{B n t^{2}}{A} \text {. }
\end{aligned}
$$

Setting $b=0$ in the above nonaxisymmetric coefficients gives the axisymmetric radiating Kerr metric of (31).

\section{References}

[1] I. Robinson and J. R. Robinson, "Vacuum metrics without symmetry," International Journal of Theoretical Physics, vol. 2, pp. 231-242, 1969.

[2] I. Robinson, A. Schild, and H. Strauss, "A generalized ReissnerNordström solution," International Journal of Theoretical Physics, vol. 2, no. 3, pp. 243-245, 1969.

[3] P. C. Vaidya, "The external field of a radiating star in general relativity," Current Science, vol. 12, no. 6, p. 183, 1943.

[4] D. Kramer, "Gravitational field of a rotating radiating source," in Proceedings of the Tezisy 3rd Soviet gravitation conference, $\mathrm{p}$. 321, Yerevan, USSR, 1972.

[5] P. C. Vaidya and L. K. Patel, "Radiating Kerr metric," Physical Review D, vol. 7, no. 12, pp. 3590-3593, 1973.

[6] R. P. Kerr, "Gravitational field of a spinning mass as an example of algebraically special metrics," Physical Review Letters, vol. 11, no. 5, pp. 237-238, 1963.

[7] E. Herlt, "Kerr-Schild-Vaidya fields with axial symmetry," General Relativity and Gravitation, vol. 12, no. 1, pp. 1-7, 1980.

[8] G. C. Debney, R. P. Kerr, and A. Schild, "Solutions of the Einstein and Einstein-Maxwell equations," Journal of Mathematical Physics, vol. 10, no. 10, pp. 1842-1854, 1969.

[9] J. Lewandowski and P. Nurowski, "Algebraically special twisting gravitational fields and CR structures," Classical and Quantum Gravity, vol. 7, no. 3, Article ID 007, pp. 309-328, 1990. 
[10] J. Tafel, P. Nurowski, and J. Lewandowski, "Pure radiation field solutions of the Einstein equations," Classical and Quantum Gravity, vol. 8, pp. L83-L88, 1991.

[11] J. Lewandowski, P. Nurowski, and J. Tafel, "Algebraically special solutions of the Einstein equations with pure radiation fields," Classical and Quantum Gravity, vol. 8, no. 3, article no. 009, pp. 493-501, 1991.

[12] A. M. Grundland and J. Tafel, "Group invariant solutions to the Einstein equations with pure radiation fields," Classical and Quantum Gravity, vol. 10, no. 11, article no. 016, pp. 23372345, 1993.

[13] I. Robinson and A. Trautman, "Some spherical gravitational waves in general relativity," Proceedings of the Royal Society A, vol. 265, pp. 463-473, 1962.

[14] D. Kramer and U. Hähner, "A rotating pure radiation field," Classical and Quantum Gravity, vol. 12, no. 9, article no. 015, pp. 2287-2296, 1995.

[15] H. Stephani, "A method to generate algebraically special pure radiation field solutions from the vacuum," Journal of Physics A, vol. 12, no. 7, article no. 021, pp. 1045-1049, 1979.

[16] H. Stephani, D. Kramer, M. A. H. MacCallum, C. Hoenselaers, and E. Herlt, Exact Solutions of Einstein's Field Equations, Cambridge University Press, Cambridge, UK, 2003.

[17] C. W. Misner, K. S. Thorne, and J. A. Wheeler, Gravitation, W. H. Freeman and Co., San Franscisco, Calif, USA, 1973.

[18] J. M. Cohen, "Angular momentum and the Kerr metric," Journal of Mathematical Physics, vol. 9, pp. 905-906, 1968. 

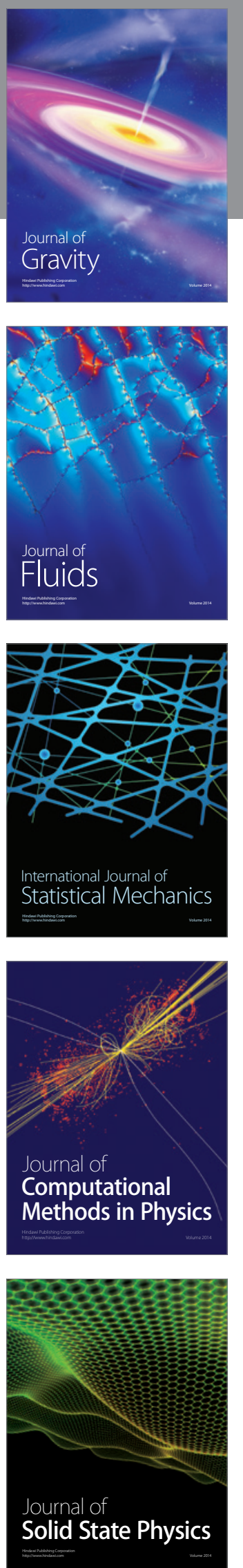

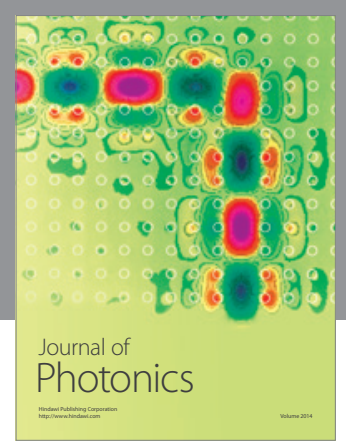

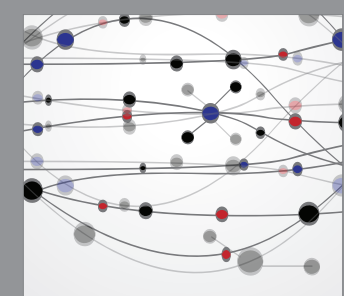

The Scientific World Journal
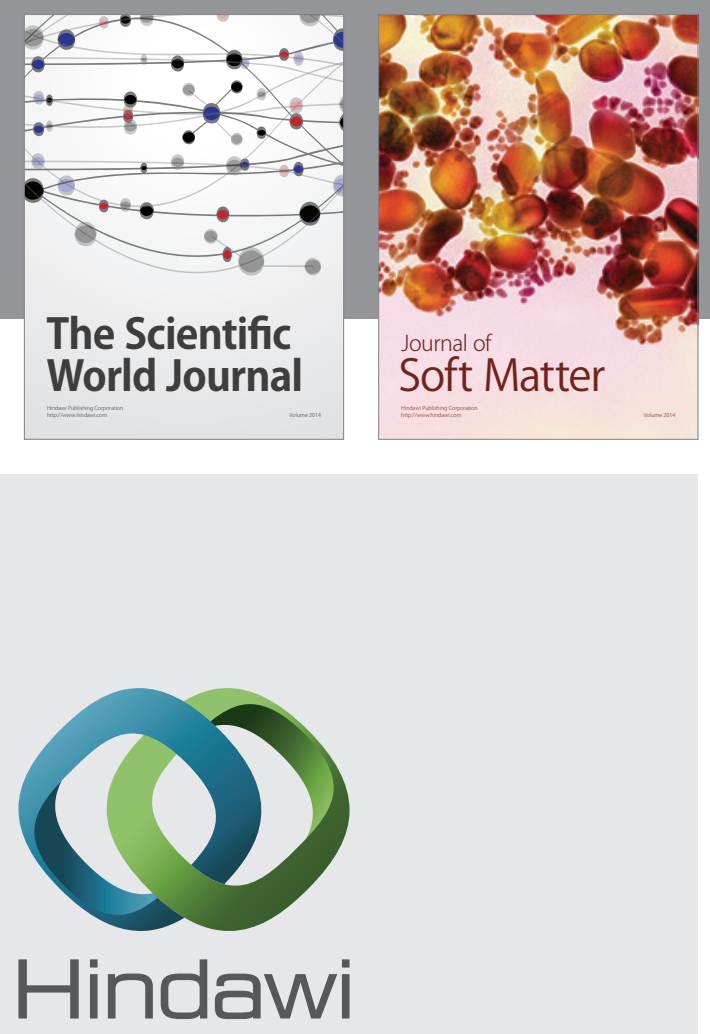

Submit your manuscripts at

http://www.hindawi.com
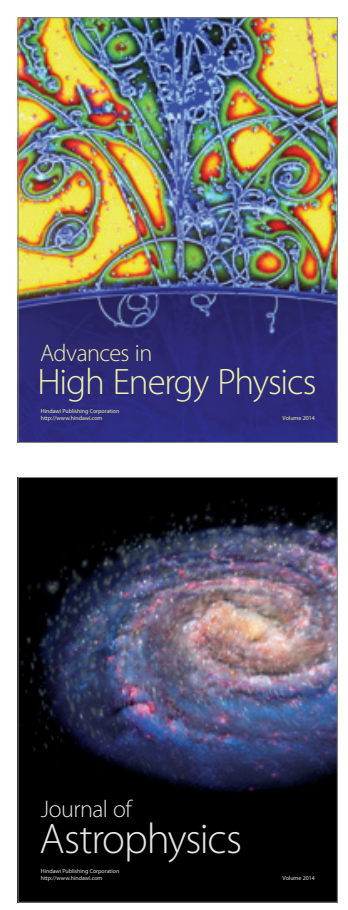
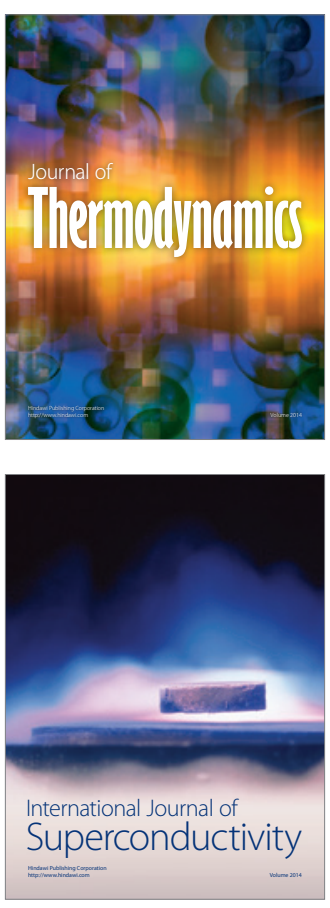
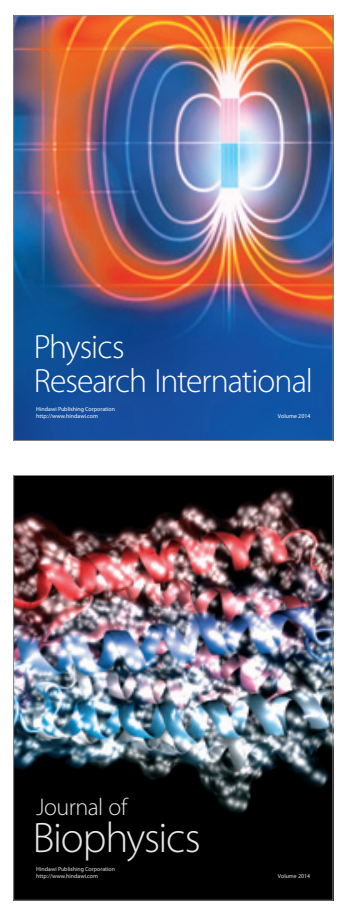
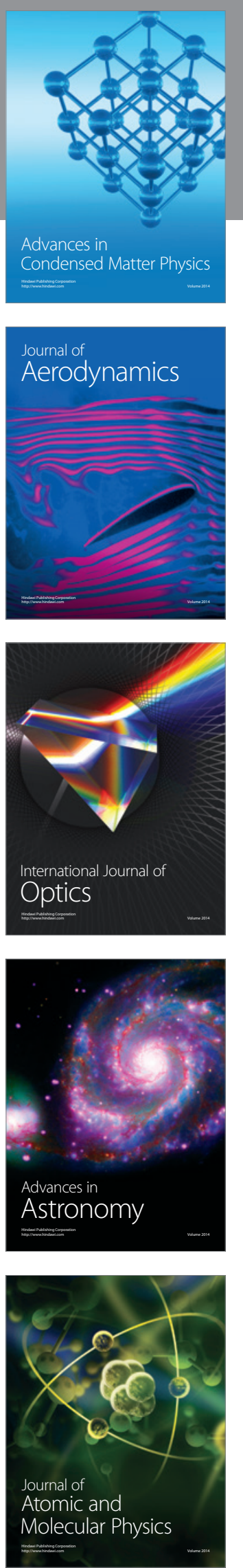